\title{
Functional Modes of Proteins Are among the Most Robust
}

\author{
S. Nicolay and Y.-H. Sanejouand \\ Laboratoire de Physique, Ecole Normale Supérieure, 46 allées d Italie, 69364 Lyon Cedex 07, France
}

(Received 14 July 2005; published 24 February 2006)

\begin{abstract}
It is shown that a small subset of modes which are likely to be involved in protein functional motions of large amplitude can be determined by retaining the most robust normal modes obtained using different protein models. This result should prove helpful in the context of several applications proposed recently, like for solving difficult molecular replacement problems or for fitting atomic structures into lowresolution electron density maps. It may also pave the way for the development of methods allowing us to predict such motions accurately.
\end{abstract}

DOI: 10.1103/PhysRevLett.96.078104

PACS numbers: 87.15.He, 46.40.-f, 87.15.-v

For two-domain proteins, it is well known that a few low-frequency normal modes can provide a fair description of their large amplitude motion upon ligand binding [1-3]. Recently, it has been shown that this is also true for proteins with complex architectures [4-8], as long as their functional motion is a collective one, i.e., if it concerns large parts of the structure [9-11]. For instance, a single mode of the $T$ form of hemoglobin is enough to describe accurately its conformational change upon oxygen binding [5].

This result has been successfully applied for exploiting fiber diffraction data $[12,13]$, solving difficult molecular replacement problems $[14,15]$, or fitting atomic structures into low-resolution electron density maps [15-17]. The principle of these applications is to perturb a known structure along its low-frequency modes so as to get a deformed structure that is consistent with low-resolution biophysical data, which are obtained after the protein has undergone some large amplitude conformational change. It was also shown that when variations of a few key distances are known, through spectroscopic measurements, for instance, it is possible, using linear response theory, to identify which modes are the most involved in the conformational change $[18,19]$. However, if such experimental data are missing, it is difficult to guess which low-frequency modes are the functional ones. Hereafter, we show that they are among the most robust ones, i.e., among the most conserved modes when different models are considered. The robustness of the functional modes was recognized when it was shown that they can be obtained [9-11] with simple protein descriptions, like elastic network (EN) models [20-23]. Herein, this property is used so as to identify them.

First, standard normal modes were calculated for a set of five proteins of different sizes and architectures after preliminary energy minimization. The CHARMM program [24] was used, with the EEF1.1 implicit solvent model [25], as done in recent studies performed at this level of detail [26]. Then, for each energy-minimized structure, low-frequency normal modes were calculated with the all-atom EN model proposed by Tirion [21], where the many-parameters em- pirical energy function $E_{p}$ used in programs like CHARMM is replaced by:

$$
E_{p}=\sum_{d_{i j}^{0}<R_{c}} C\left(d_{i j}-d_{i j}^{0}\right)^{2}
$$

where $d_{i j}$ is the distance between atoms $i$ and $j, d_{i j}^{0}$ being their distance in the studied structure. The strength of the potential $C$ is a constant assumed to be the same for all interacting pairs. It is required only in order to define units. As done in previous studies [14], $R_{c}$, the cutoff parameter, is set to $5 \AA$.

In order to compare both sets of normal modes, $n_{i}^{\text {eff }}$, the effective number of EN modes involved in the description of standard mode $i$, is calculated as follows [27]:

$$
n_{i}^{\mathrm{eff}}=\exp \left[-\sum^{n} \alpha I_{i j}^{2} \ln \left(\alpha I_{i j}^{2}\right)\right],
$$

where $n$ is the number of EN modes taken into account ( $n=100$ herein), $I_{i j}$ being the scalar product between standard mode $i$ and EN mode $j$. The normalization factor $\alpha$ is such that: $\sum \alpha I_{i j}^{2}=1$. Thus, $n_{i}^{\text {eff }}$ gives the effective number of nonzero $I_{i j}^{2}$. It ranges from 1 to $n$. As shown in Fig. 1, for each protein considered, a few standard modes, with low ranking, can be described accurately with less than 5-6 EN modes. For a given protein, the set of such modes defines a subspace which is robust, that is, well conserved when modes are calculated with very different models.

Next, such robust modes were sought for, using this time two different EN models. In both cases, as often done [9$11,20,22,23,28]$, only $C_{\alpha}$ atoms are kept. In the first model, pairs of interacting neighbors are determined according to a distance-cutoff criterion. Setting $R_{c}$ to a value lower than 8-10 A splits the elastic network into several independent ones and the number of zero-frequency modes becomes larger than 6. To avoid this artifact, values of $10-15 \AA$ have been used $[9,19,28]$. For adenylate kinase, with $R_{c}=$ $12 \AA, n_{c}$, the average number of interacting neighbors per $C_{\alpha}$ atom is $25 \pm 7$, ranging from 10 to 42 , as a function 


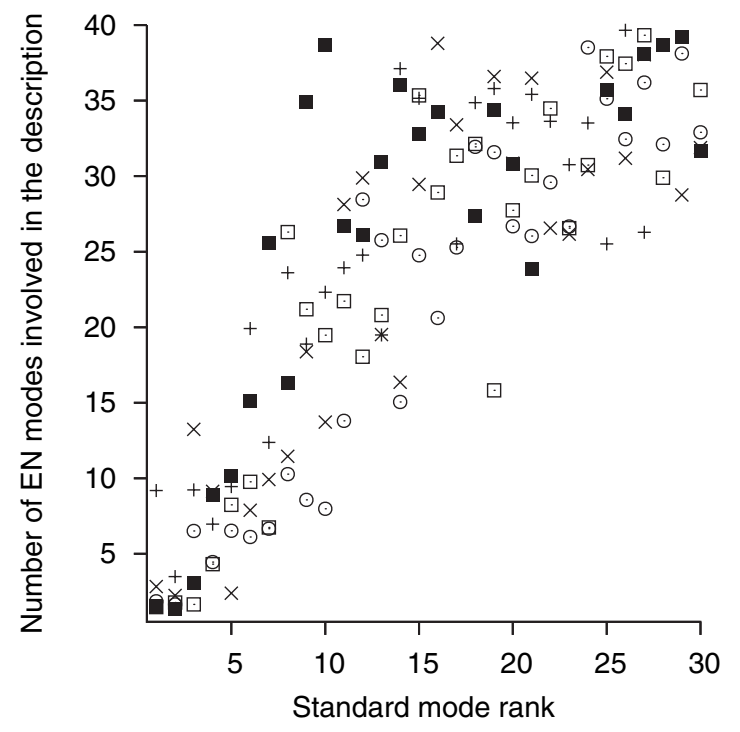

FIG. 1. Effective number of EN normal modes involved in the description of each standard mode of five proteins. Cross: Lysozyme T4 (pdb code 1781). Plus: Adenylate kinase (4ake). Open square: Glutamin binding protein (1ggg). Filled square: LAO binding protein (2lao). Open circle: DNA polymerase $\beta$ (1bpx). Modes are ranked according to increasing frequencies.

of the degree of burial of the amino acid in the protein interior.

We designed the second EN model so as to keep $n_{c}$ as constant as possible from one amino acid to the other. To do so, the following algorithm was used. First, all pairs of $C_{\alpha}$ atoms are sorted, according to their distance. Then, starting from the atom pair separated by the largest distance, they are removed one after the other, unless one atom of the pair has already $n_{c}$ neighbors. With this algorithm, setting $n_{c}=10$, the average distance between pairs of interacting neighbors is $6.2 \pm 1.8 \AA$, close to typical cutoff values used for determining contacts between amino acids in proteins [29]. In the case of adenylate kinase, $n_{c}$ can be set to a value as low as 7 (see Fig. 2) without splitting the network into independent ones.

As done above, normal modes obtained with both EN models were compared, seeking for robust ones, for a set of

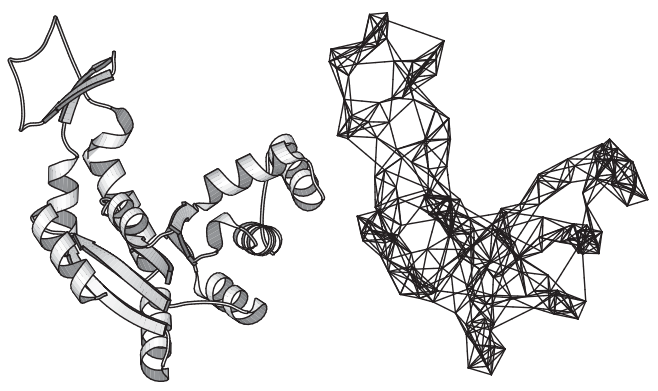

FIG. 2. The open form of adenylate kinase (pdb code 4ake). Right: the new $N$-neighbors elastic network model introduced in this study. Most $C_{\alpha}$ atoms are linked to seven neighbors $(N=$ 7). Drawn with Molscript [31].
22 proteins considered in previous studies performed with the distance-cutoff criterion $[9,10,14]$. Like in the case of all-atom models, modes are considered to be robust whenever $n_{i}^{\text {eff }} \leq 6$. Statistics of the number of robust modes found for all studied proteins are shown in Fig. 3. In most cases, the number of robust modes is four or less. In only three cases, it is larger than 7. Not surprisingly, they correspond to proteins with large sizes (more than 500 amino acids) and complex architecture, like the DNA polymerase of bacteriophage RB69 (pdb code 1ih7), which is the protein of our data set with the largest number of robust modes (eleven). In four cases, no robust mode is found. Interestingly, the known conformational change of these proteins, namely, tyrosine phosphatase, triose phosphate isomerase, che Y, and HIV-1 protease (pdb codes 1yts, 3tim, 3chy, $1 \mathrm{hhp}$, respectively), is a small amplitude one, with a $C_{\alpha}$ root-mean-square displacement (rmsd) of $1.5 \AA$ at most.

Then, it was checked that robust modes yield accurate descriptions of protein functional motions. To do so, $Q_{d}$, the quality of the motion description is calculated as follows $[5,10]$ :

$$
Q_{d}=100 \sum^{n} I_{i d}^{2}
$$

where $n$ is the number of modes taken into account in the description and $I_{i d}$ is the scalar product between mode $i$

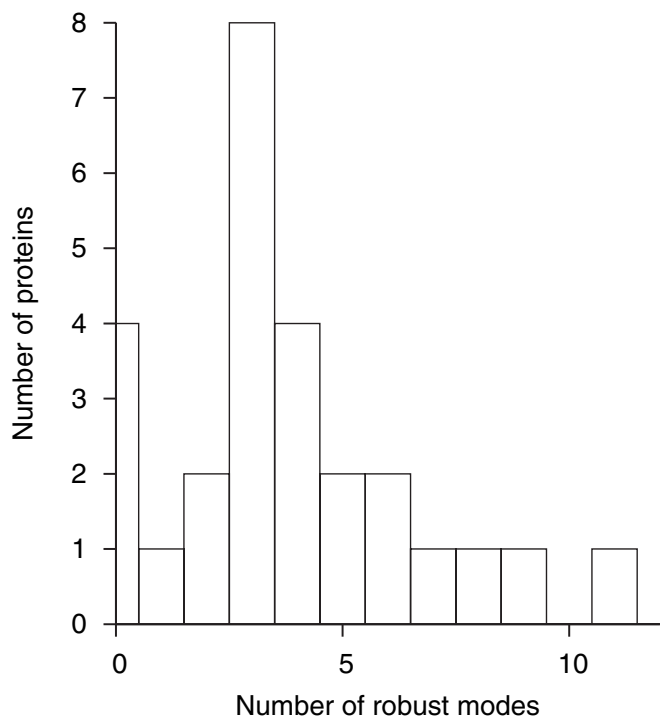

FIG. 3. Number of robust normal modes found by comparing modes obtained with different protein models. For a first set of five proteins, standard modes were compared to modes obtained with an all-atom EN model. For a second set of 22 proteins (pdb codes 1781, 1a3n, 1aro, 1bpx, 1cb6, 1ggg, 1hhp, 1hil, 1ih7, 1omp, 1usg, 1yts, 2ktq, 3chy, 3tim, 3tms, 4ake, 4tgl, 5csc, $5 \mathrm{dfr}$, 8adh, 9aat), modes obtained with two different $C_{\alpha}$-EN models were compared. Modes are considered to be robust when they can be described accurately with at most six modes obtained with the other model. 
and the direction of the conformational change observed by crystallographers. Note that $Q_{d}=100 \%$ when all modes are included in the description, since they form a complete basis set.

As shown in Fig. 4, the conformational change of lactoferrin upon ligand (iron) binding can be described accurately $\left(Q_{d}\right.$ over $\left.85 \%\right)$ as a linear combination of the seven lowest-frequency modes of the open form (pdb code 1cb6). Interestingly, all seven modes are found to be robust. In Fig. 5, $Q_{d}$ is given as a function of the amplitude of the functional motion of each protein considered when $n=$ 100 normal modes or when only the robust ones are taken into account in the description. For most proteins with small amplitude motions, i.e., of less than $2-3 \AA$ of rmsd, robust modes fail to capture any information about the nature of the known conformational change, while in several cases some information is indeed present in the normal modes. For instance, as mentioned above, for HIV1 protease no robust mode is found, although a single EN mode is enough for describing 50\% of its conformational change upon ligand binding [9]. If two other EN modes are added to the description, $Q_{d}$ can reach a value of $77 \%$ (with $n=100, Q_{d}=89 \%$ ).

On the other hand, when considering proteins with large amplitude motions, the description of the conformational change with robust modes is almost as accurate $\left(Q_{d}\right.$ over $75 \%$ ) as when $n=100$ normal modes are taken into account. The only counter example is adenylate kinase, whose rmsd upon ligand binding is $5.3 \AA$. As a matter of fact, when standard normal modes of adenylate kinase are compared to all-atom EN ones, only a single robust mode is found (see Fig. 1), and it is not involved in the conforma-

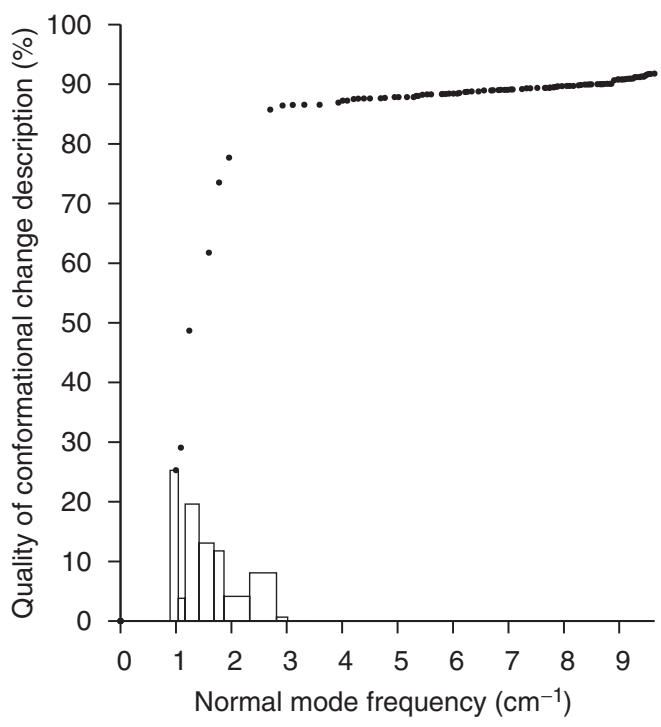

FIG. 4. Quality of the description of the closure motion of lactoferrin upon ligand binding, as a function of the number of low-frequency normal modes (black points) considered. Boxes: contribution of each robust mode to the description. tional change $\left(Q_{d}=4 \%\right)$. However, using the pair of $C_{\alpha}$-EN models, six robust modes are found and they allow for an almost perfect description of the conformational change $\left(Q_{d}=91 \%\right)$.

Of course, when using all-atom models, more robust modes can be obtained by raising the robustness criterion. In the case of adenylate kinase, if a given mode is said robust whenever $n_{i}^{\text {eff }} \leq 10$, then five robust modes are found. However, it is still not enough $\left(Q_{d}=73 \%\right)$ for describing its conformational change as well as with robust modes obtained using $C_{\alpha}$-EN models. Raising the robustness criterion so as to obtain six robust modes does not change significantly the quality of the description $\left(Q_{d}=\right.$ $77 \%$ ). As a matter of fact, robust modes obtained using allatom models always yield poorer description of protein functional motions than when using simpler models, in which only $C_{\alpha}$ atoms are kept (open circles are below open squares in Fig. 5). This is likely due to the fact that standard normal mode analysis requires a preliminary energy minimization, during which the structure is significantly distorted, while normal mode analysis of EN models does not, as illustrated by the case of DNA polymerase $\beta$. For this protein, when the $C_{\alpha}$-EN models are built using the crystal structure (pdb code $1 \mathrm{bpx}$ ), seven robust modes are found, which are able to describe accurately $\left(Q_{d}=\right.$ $84 \%$ ) the conformational change upon nucleotide binding. However, when they are built using the energy-minimized structure, only three robust modes are found, which are not able to describe the conformational change $\left(Q_{d}=21 \%\right)$ much better than the three ones obtained using all-atom

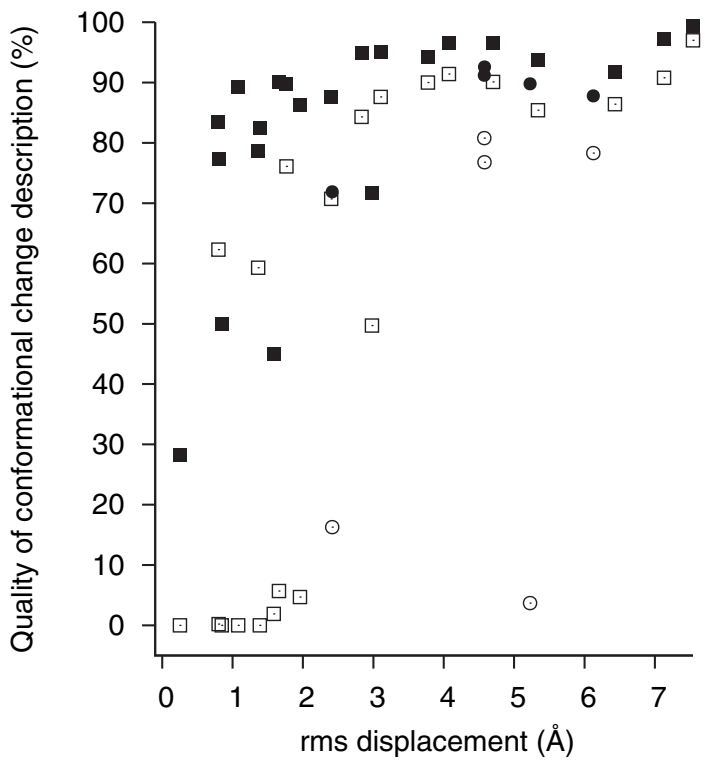

FIG. 5. Quality of the description of protein functional motions with 100 low-frequency modes (filled symbols) or with only the robust ones (open symbols), as a function of the amplitude of the motion. Five proteins were studied at the all-atom level (circles) and the other ones at the amino-acid level (squares). 
models $\left(Q_{d}=16 \%\right)$. In that case, the distortion due to energy minimization is large ( $\mathrm{rmsd}=2.5 \AA$ ), as a consequence of the removal of the ligand, a 16 base pair DNA, prior to the calculation. However, protein distortions upon energy minimization are usually not that large $(\mathrm{rmsd}=$ 1-2 $\AA$ ) and further work is required in order to fully clarify the origin of this counterintuitive, slight but systematic, effect.

In the present study, modes obtained with different protein models were compared. For most protein cases, several robust modes are found, confirming results obtained previously $[9,20-23,30]$, namely, that the lowestfrequency modes are little sensitive to details in the protein description. Since in the case of current EN models atomatom interactions are defined with a distance-cutoff criterion, this can be explained in two different ways. First, robust modes may capture information about the protein mass distribution in space. Second, they may capture information about the rigidity of the protein in the vicinity of each amino-acid residue. Indeed, with a distance-cutoff criterion, amino acids in the protein interior are more rigid (more neighbors) than those on the surface (less neighbors). So, we designed a novel $C_{\alpha}$-EN model whose main raison d'être was to decide between these two possibilities. In this model, each $C_{\alpha}$ atom has a given number of interacting neighbors and rigidity is fairly constant from one point of a protein to another. When modes obtained with this model are compared to those obtained with a $C_{\alpha}$-EN model based on the distance-cutoff criterion, robust modes are also found. This means that they are also not sensitive to the distribution of rigidity in the protein.

Moreover, we have shown that these robust modes are likely to be involved in protein functional motions, at least when the functional motion is a large amplitude one (rmsd $\geq 2-3 \AA$ ). This result should prove helpful in the context of applications like those mentioned in the introduction, since they all concern large amplitude conformational changes [14-17].

This result could also pave the way for the development of methods allowing to predict such motions accurately, i.e., to predict their amplitude, since exploring a subspace of small dimensionality (three or four in most cases considered) should be enough for finding conformations close to functional ones. Interestingly, seeking for robust modes could also indicate whether a given protein can exhibit a large amplitude functional motion or not. Indeed, the functional motions of the proteins found to have no robust mode are small amplitude ones.
[1] J.A. McCammon, B.R. Gelin, M. Karplus, and P. Wolynes, Nature (London) 262, 325 (1976).

[2] W. Harrison, Biopolymers 23, 2943 (1984).

[3] B. R. Brooks and M. Karplus, Proc. Natl. Acad. Sci. U.S.A. 82, 4995 (1985).

[4] O. Marques and Y.-H. Sanejouand, Proteins 23, 557 (1995).

[5] D. Perahia and L. Mouawad, Computers and Chemistry 19 , 241 (1995).

[6] J. Ma, Structure 13, 373 (2005).

[7] I. Bahar and A. Rader, Curr. Opin. Struct. Biol. 15, 586 (2005).

[8] C. Chennubhotla, A. Rader, L. Yang, and I. Bahar, Physical Biology 2, S173 (2005).

[9] F. Tama and Y.-H. Sanejouand, Protein Eng. 14, 1 (2001).

[10] M. Delarue and Y.-H. Sanejouand, J. Mol. Biol. 320, 1011 (2002).

[11] W. G. Krebs, V. Alexandrov, C.A. Wilson, N. Echols, H. Yu, and M. Gerstein, Proteins 48, 682 (2002).

[12] M. Tirion, D. ben Avraham, M. Lorenz, and K. Holmes, Biophys. J. 68, 5 (1995).

[13] Y. Wu and J. Ma, Biophys. J. 86, 116 (2004).

[14] K. Suhre and Y.-H. Sanejouand, Acta Crystallogr. Sect. D 60, 796 (2004).

[15] M. Delarue and P. Dumas, Proc. Natl. Acad. Sci. U.S.A. 101, 6957 (2004).

[16] F. Tama, O. Miyashita, and C. L. Brooks, III, J. Mol. Biol. 337, 985 (2004).

[17] K. Hinsen, N. Reuter, J. Navaza, D. L. Stokes, and J. J. Lacapere, Biophys. J. 88, 818 (2005).

[18] M. Ikeguchi, J. Ueno, M. Sato, and A. Kidera, Phys. Rev. Lett. 94, 078102 (2005).

[19] W. Zheng and B.R. Brooks, Biophys. J. 88, 3109 (2005).

[20] T. Haliloglu, I. Bahar, and B. Erman, Phys. Rev. Lett. 79, 3090 (1997).

[21] M. Tirion, Phys. Rev. Lett. 77, 1905 (1996).

[22] I. Bahar, A. R. Atilgan, and B. Erman, Folding Des. 2, 173 (1997).

[23] K. Hinsen, Proteins 33, 417 (1998).

[24] B. R. Brooks, R. E. Bruccoleri, B. D. Olafson, D. J. States, S. Swaminathan, and M. Karplus, J. Comput. Chem. 4, 187 (1983).

[25] T. Lazaridis, Proteins 52, 176 (2003).

[26] G. Li and Q. Cui, Biophys. J. 86, 743 (2004).

[27] R. Bruschweiler, J. Chem. Phys. 102, 3396 (1995).

[28] A. Atilgan, S. Durell, R. Jernigan, M. Demirel, O. Keskin, and I. Bahar, Biophys. J. 80, 505 (2001).

[29] S. Miyazawa and R. Jernigan, J. Mol. Biol. 256, 623 (1996).

[30] M. Lu and J. Ma, Biophys. J. 89, 2395 (2005).

[31] P. Kraulis, J. Appl. Crystallogr. 24, 946 (1991). 